\title{
Prometheus' predicament: How to address contemporary left ventricular assist devices in patients with liver dysfunction
}

\author{
Elena Sandoval, MD, Julius A. Carillo, MD, and Steve K. Singh, MD
}

From the Division of Transplant and Assist Devices, Baylor College of Medicine, Houston, Tex.

Disclosures: Authors have nothing to disclose with regard to commercial support.

Received for publication Sept 3, 2015; accepted for publication Sept 5, 2015; available ahead of print Oct 15, 2015 .

Address for reprints: Steve K. Singh, MD, 6770 Bertner Ave, C355A, Houston, TX 77030 (E-mail: steve.singh@ bcm.edu).

J Thorac Cardiovasc Surg 2016;151:236-7

0022-5223/\$36.00

Copyright (c) 2016 by The American Association for Thoracic Surgery

http://dx.doi.org/10.1016/j.jtcvs.2015.09.048

The impact of chronic congestive heart failure is without debate, affecting more than 5 million people in the United States and tens of millions worldwide. The sequelae of end-stage heart failure include potential gradual declines in multiple organs. The impact on the liver is critical in the decision for heart transplant or left ventricular assist device (LVAD) therapies.

The retrospective single-center review by Sargent and colleagues ${ }^{1}$ in this issue of the Journal addresses important and common questions when selecting patients for LVAD therapy: (1) Which patients with liver dysfunction would benefit from LVAD therapy? (2) What degree of liver dysfunction will halt or reverse with LVAD therapy?

In heart failure, liver dysfunction is due to a dual mechanism: poor organ perfusion leading to ischemic changes and right ventricular failure responsible for hepatic venous congestion. ${ }^{2}$ Early stages of these changes are reversible; however, long-term congestive hepatopathy may lead to irreversible damage through fibrosis changes, continuing to "cardiac cirrhosis." LVAD therapy has become a standard treatment for end-stage heart failure. Although early and overall clinical outcomes after LVAD placement are impacted by end-organ function, the benefits to cardiacrelated liver dysfunction may allow reversal of damage for ongoing destination therapy or eligibility for heart transplant (vs a heart-liver transplant associated with greater morbidity).

Sargent and colleagues ${ }^{1}$ present a notable series of 233 patients with contemporary continuous-flow LVADs, demonstrating similar short-term survival in 14 patients with periportal fibrosis on liver biopsy who received LVADs as in patients without fibrosis who received LVADs. The main strength of Sargent and colleagues' work ${ }^{1}$ is the use of criterion standard of liver biopsy to characterize the degree of histologic lesion and liver damage. The sample size is acknowledged to be a limitation; however, few studies have significantly greater biopsy data. Another limitation is the inclusion of only periportal fibrosis

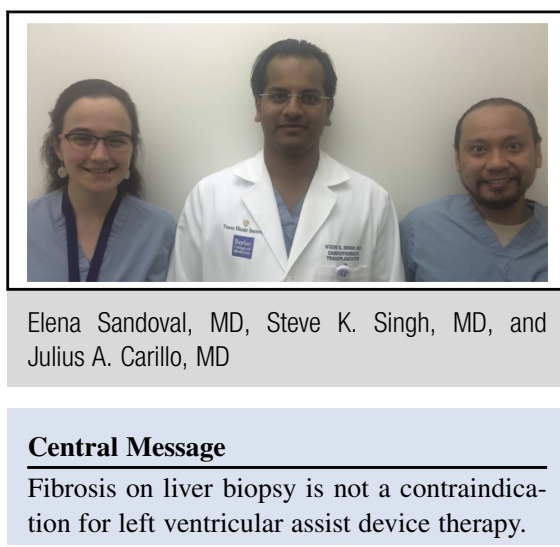

See Article page 230.

See Editorials page 10 and 13.

(Meta-analysis of Histological Data in Viral Hepatitis classification), which is equivalent to stage 1 , or mild fibrosis, in the more commonly used International Association for Study of the Liver classification. ${ }^{3,4}$ This population thus does not include any cases of moderate or advanced liver dysfunction. Clinically and biochemically the levels of transaminases, bilirubin, and so on, are similar between the groups in the fibrosis and nonfibrosis groups, suggesting that the degree of liver dysfunction was mild.

At our institution, Demirozu and colleagues have published a cohort of 338 patients receiving continuous-flow LVADs, 23 of whom had advanced liver dysfunction. This was defined as having transaminases more than 5 times normal, serum bilirubin more than 3 times normal, or liver biopsy (in 9 patients). Liver biopsy showed periportal fibrosis (International Association for Study of the Liver stage 1 fibrosis), along with periportal septae (stage 2, or moderate, fibrosis), and advanced centrilobar necrosis. Biopsy included measurements of hepatic venous gradients. Mean Model for End-Stage Liver Disease (MELD) score was 21. Despite such advanced liver dysfunction, the LVAD liver dysfunction versus no liver dysfunction cohorts had similar 1-year survivals. The liver dysfunction cohort also showed marked improvement in follow-up biochemistry values.

There are more data on the biochemical markers and the MELD score and outcomes after LVAD placement. Deo and colleagues ${ }^{6}$ showed a correlation between MELD score and 
survival in patients with LVADs undergoing first-time surgery. Yang and coworkers ${ }^{7}$ showed that MELD excluding the international normalized ratio (MELD-XI) is a valuable tool in the stratification of risk for patients with LVADs; they established a cut-off at a preoperative MELD-XI of 17, showing worse results in patients with higher MELD-XI scores. Furthermore, their same work showed MELD-XI to be important in the assessment of liver recovery after LVAD insertion, showing better posttransplant survival in those patients whose MELD-XI scores improved during LVAD support.

Although LVAD technology and techniques are improving, the success of contemporary continuous-flow LVAD therapy remains tied to patient selection. With respect to concomitant liver dysfunction, Sargent and colleagues ${ }^{1}$ present excellent 1 -year survivals in patients with early periportal fibrosis. There is a paucity of data in the literature; however, it suggests that liver fibrosis is not an absolute contraindication for LVAD therapy. More study is warranted on the impact of criterion standard liver biopsy characteristics (histologic features and venous pressures) and LVAD outcomes. Easily acquired biochemical markers of liver disease and scores such as MELD and MELD XI, need robust validation as prognostic tools for risk in the LVAD population.

Prometheus, meaning "forethought," was the Greek deity who was the creator of mankind, and gifted mankind with fire stolen from Mount Olympus. As eternal punishment, he was chained by Zeus to a rock to have his liver eaten daily by an eagle, only to be regenerated at night because of his immortality. Although we wish our patients had this regenerative ability, it is more apt to consider Prometheus for its meaning of forethought when we consider our patient selection for LVADs.

\section{References}

1. Sargent JE, Dardas TF, Smith JW, Pal JD, Cheng RK, Masri SC, et al. Periporta fibrosis without cirrhosis does not affect outcomes following continuous flow ventricular assist device implantation. J Thorac Cardiovasc Surg. 2016;151: 230-5.

2. Wadia Y, Etheridge W, Smart F, Wood RP, Frazier OH. Pathophysiology of hepatic dysfunction and intrahepatic cholestasis in heart failure and after left ventricular assist device support. J Heart Lung Transplant. 2005;24:361-70.

3. Bedossa P, Poynard T. An algorithm for the grading of activity in chronic hepatitis C. The METAVIR Cooperative Study Group. Hepatology. 1996;24:289-93.

4. Desmet VJ, Gerber M, Hoofnagle JH, Manns M, Scheuer PJ. Classification of chronic hepatitis: Diagnosis, grading and staging. Hepatology. 1994;19: 1513-20.

5. Demirozu ZT, Hernandez R, Mallidi HR, Singh SK, Radovancevic R, Segura AM, et al. Heart Mate II Left ventricular assist device implantation in patients with advanced hepatic dysfunction. J Card Surg. 2014;29:419-23.

6. Deo SV, Daly RC, Altarabsheh SE, Hasin T, Zhao Y, Shah IK, et al. Predictive value of the model for end-stage liver disease score in patients undergoing left ventricular assist device implantation. ASAIO J. 2013;59:57-62.

7. Yang JA, Kato TS, Shulman BP, Takayama H, Farr M, Jorde UP, Mancini DM, et al. Liver dysfunction as a predictor of outcomes in patients with advanced heart failure requiring ventricular assist device support: use of the Model of End-stage Liver Disease (MELD) and MELD eXcluding INR (MELD-XI) scoring system. J Heart Lung Transplant. 2010;31:601-10. 